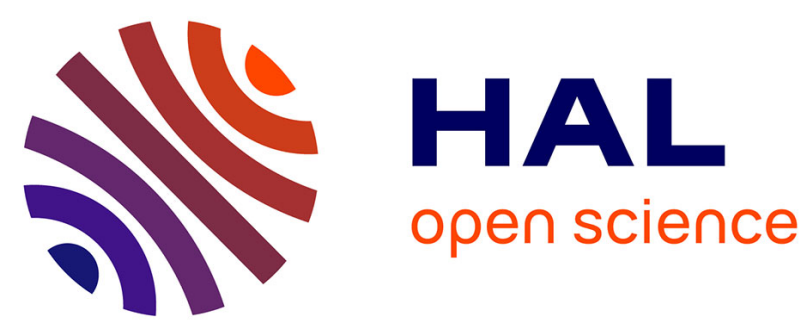

\title{
Interoperable Infrastructure and Implementation of a Health Data Model for Remote Monitoring of Chronic Diseases with Comorbidities
}

P. Finet, B. Gibaud, Olivier Dameron, Régine Le Bouquin Jeannès

\section{- To cite this version:}

P. Finet, B. Gibaud, Olivier Dameron, Régine Le Bouquin Jeannès. Interoperable Infrastructure and Implementation of a Health Data Model for Remote Monitoring of Chronic Diseases with Comorbidities. Innovation and Research in BioMedical engineering, 2018, 39 (3), pp.151 - 159. 10.1016/j.irbm.2018.03.003 . hal-01815338

\section{HAL Id: hal-01815338 \\ https://hal.science/hal-01815338}

Submitted on 14 Jun 2018

HAL is a multi-disciplinary open access archive for the deposit and dissemination of scientific research documents, whether they are published or not. The documents may come from teaching and research institutions in France or abroad, or from public or private research centers.
L'archive ouverte pluridisciplinaire HAL, est destinée au dépôt et à la diffusion de documents scientifiques de niveau recherche, publiés ou non, émanant des établissements d'enseignement et de recherche français ou étrangers, des laboratoires publics ou privés. 


\title{
Interoperable Infrastructure and Implementation of a Health Data Model for Remote Monitoring of Chronic Diseases with Comorbidities
}

\author{
P. Finet ${ }^{a}$ B. Gibaud ${ }^{b, c}$, O. Dameron ${ }^{c, d}$ and R. Le Bouquin Jeannès ${ }^{b, c}$ \\ ${ }^{a}$ Centre Hospitalier Intercommunal Alençon-Mamers, Alençon, F-61000 France \\ ${ }^{\mathrm{b}}$ INSERM, U 1099, Rennes, F-35000 France \\ ${ }^{\mathrm{c}}$ Université de Rennes 1, LTSI, Rennes, F-35000, France \\ ${ }^{d}$ IRISA, Université de Rennes 1, F-35042 Rennes, France
}

Corresponding author:

Email: philippe-finet@orange.fr

\section{Highlights}

- To adopt a common health data model compatible with chronic diseases and their comorbidities.

- To implement an interoperable telemedicine platform.

- Data structure of our health data model based on HL7 CDA (Clinical Document Architecture).

- Context-aware generic model suitable for most acquisition devices.

\section{Graphical abstract}

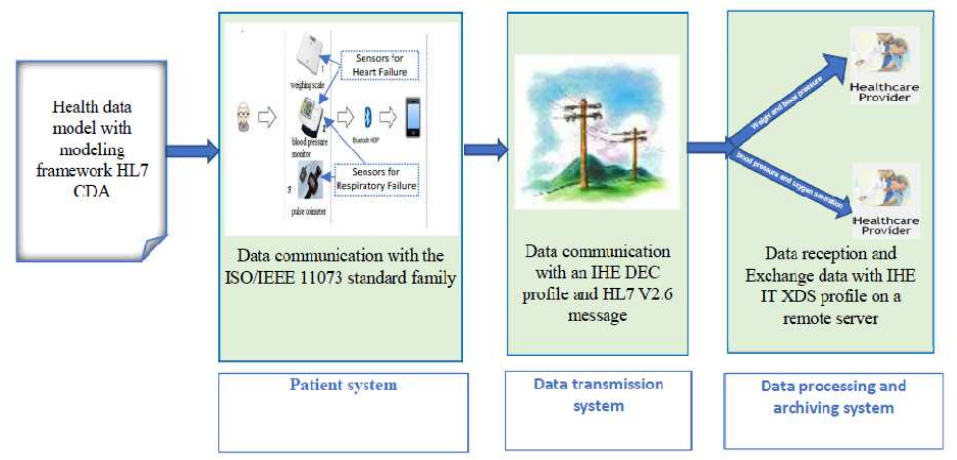

\begin{abstract}
-
Background: The future increase of chronic diseases justifies the development of telemedicine for following up patients outside of the hospital. However, current telemedicine applications are disease-specific whereas chronic diseases are often associated with comorbidities.
\end{abstract}

Methods: We show that the use of interoperability standards for telemedicine systems makes it possible to build a telemedicine platform (with several medical devices) that can be shared between several diseases workflows. To remotely follow up the patient's vital signs, health professionals need to access to the context description and the relevant background information. The implementation of a health data model that meets the needs of practitioners seems to be an appropriate solution to this problem.

Results: To validate our architecture model of telemedicine application, we conducted various communication tests of vital signs to prove the interoperability of the patient system. The second experiment, we have developed one HL7 CDA document which collects several vital signs to a medical data exchange system among healthcare providers. This led us to propose a telemedicine application model, which is not only in conformity with the Health Information Systems Interoperability Framework (HIS-IF) of the "Agence des Systèmes d'Information Partagés de Santé" (ASIP), but also constitutes a proposed extension of this framework to the patient's home.

Keywords: Telemedicine; Interoperability; Health data model

\section{Introduction}

The ageing of the population is associated with the development of chronic diseases [1], which were responsible for 36 of the 57 million deaths worldwide in 2008 [2]. The United Nations estimated that this category is expected to reach 406 million by 2050 [3]. The aging of the population will result in an increase of the number of chronic diseases with comorbidity factors [4]. For example, compared with people who do not have diabetes, the diabetic patients have a higher risk of cardiovascular morbidity. Type 2 diabetes is associated with a two-fold to fourfold increased risk of coronary artery disease, and a twofold to eight-fold increased risk of heart failure [5]. Chronic diseases typically require regular follow-up 
and are therefore expected to have a strong impact on the medical organization of care and on the cost for society. This is further exacerbated by co-morbidities. The use of telemedicine facilitates patient follow-up outside of the hospital. Telemedicine applications can contribute to reduce healthcare costs by reducing the number of hospital consultations and transports. However, most existing telemedicine projects focus on a single chronic disease and few actors. In such cases, comorbidities are usually not explicitly considered. In fact, taking comorbidities into ac-count requires the ability to share medical data among health care information systems. For having access to care anywhere and anytime with telemedicine, it is necessary to create a fully interoperable infrastructure [6]. The expected benefits of such an architecture are two-fold: first, avoid the redundancy of medical information systems as isolated islands, and second, enable chronic disease telemedicine applications to take into account parameters from the comorbidities. After reviewing communication standards, it also seemed important to define the contents of patient data to share with the medical personnel in order to ensure that the provided information is sufficient for clinical diagnosis.

The aim of our project is to devise an interoperable telemedicine system suitable for monitoring article consists in identifying a model of data structure that meets the medical needs of remote monitoring for several chronic diseases with various medical devices. This led us to propose in a second part a new implementation of interoperable telemedicine systems which allows healthcare professionals to share patient information.

\section{Materials and method}

To analyze patient's data, health professionals need to access the context description (e.g. information about the type of examination), the important background information (e.g. the type of collected measurements from medical devices and the date and the time of the measurements) and the acquisition context (e.g. sensor characteristics). Furthermore, if ex-changed information is limited to raw data captured by patients, it is not possible to interpret it meaningfully. Patient's data are context-dependent [7], and measurement results (weigh, blood pressure, oxygen saturation oxygen, etc.) have different meanings depending on the time of measurement [7]. They cannot be properly interpreted by a remote medical staff without specific contextual information. In this case, we define a health data model available for different chronic diseases and containing the necessary information for the vital signs analyzed. Our health data model must be able to provide the medical needs for several chronic diseases. To improve our health data model, an environment should be implemented to share and exchange patient's data in order to facilitate cooperation between healthcare professionals. As HL7 Clinical Document Architecture (CDA) [8] is the appropriate standard for sharing medical documents, we decided to use its structure to build our health data model. To achieve our goal, we must communicate all data of our health data model from patient's data to the remote computer system. In this case, the telemedicine systems must be fully interoperable from the technical (between two different technology systems), syntactic (between two different communication systems) and semantic points of view.

In order to address the challenges of fully interoperable sys-tem for information sharing and sufficient context information for remote diagnosis, we propose the use of standard data ex-change formats, interoperable services and standard transport protocols to send structured data to a remote location addresses. To verify our system modeling, we perform the mapping between data of our health data model and the data models from the communication standards used. Laboratory experimentations have enabled to validate our modeling system for the following sensors: a blood pressure monitor, a pulse oximeter and a weighing scale. These three sensors allow us to obtain all possible configurations of our health data model. In fact, the weighing scale DIM model has only one numeric object, whereas the blood pressure monitor DIM model and the pulse oximeter DIM model have two numeric objects. A laboratory trial gave us the opportunity to demonstrate interoperability between the sensors and the smartphone, based on the ISO/IEEE 11073 family of standards [9]. To test the medical data exchange, we have specified a HL7 CDA compliant document structure for collecting several vital signs. We have tested this document structure with the Gazelle platform tool ${ }^{1}[10]$.

\section{Proposed telemedicine system}

\subsection{Conception of the health data model}

To define a health data model suitable for telemedicine projects, we selected an appropriate modeling framework from the international standard HL7 Clinical Document Architecture (CDA) [8]. The aim of this standard which is derived from the HL7 Reference Information Model (RIM) [8] is to share inter-operable clinical documents between healthcare professionals. This standard supports acquisition, transfer and exchange of both qualitative and quantitative data captured in a telemedicine context. The CDA R2 version includes structured elements in the document header and in the document body [11]. This type of documents may include several sections that contain human readable narrative forms or codified structures for an automatic processing. Thus, it contains health data that can be easily integrated into databases of healthcare facilities [8]. With the last version of CDA, the body text sections include

\footnotetext{
${ }^{1}$ Gazelle is an open source platform that allows a wide set of tools to test information exchange.
} 


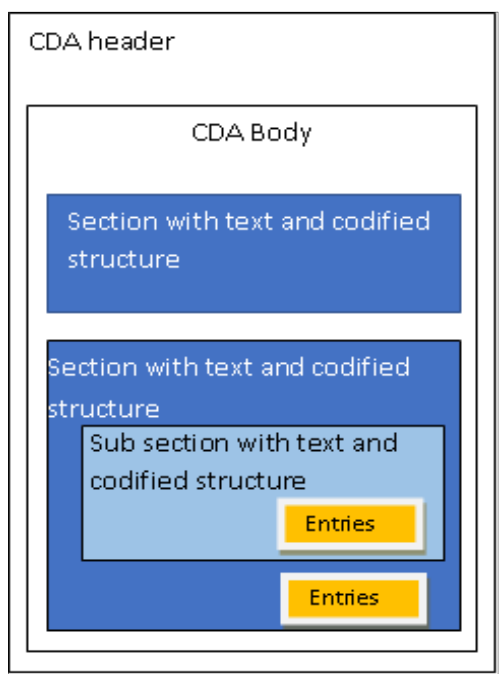

Fig. 1. CDA structure

codified entries to recover external data (Fig.1) [11]. The implementation of a CDA document requires to select an existing model that contains templates and rules sets. Templates are designed to create standardized clinical documents that are specifically intended to support clinical workflows in various use cases [12]. For our telemedicine project and our health data model, we chose a CDA document model which aims to get vital signs: the "Personal Healthcare Monitoring Report" (PHMR) [13].

This type of document contains different templates and values. For each medical device used at the patient's home, the creation of the health context model consists of describing variables, constants and codes which are present in the CDA header, in a CDA section, in a CDA subsection or in a predefined entry. To define the context of the data within the health data model, we have used information contained within section of CDA documents that contain collections of vital signs. We have re-lied, on the one hand, on implementation guides that deal with patient measurement data generated by medical devices, and on the other hand, on the Health Information Systems Interoperability Framework (HIS-IF) by ASIP. The selected implementation guides are the "Continuity of Care Document" (CCD) [14], the PHMR [13], as well as the document provided by ASIP "CI-SIS Couche Contenu Volet Structuration_Minimale" [15]. These documents identified the relevant information related to vital signs: the measurement descriptions, the various nomenclature codes, the codes for measurement units and the date and time (cf. Fig.2).

In order to include all the data necessary in the health data model, we need sensor data identification to ensure traceability. Our health data model also contains a section with sensor information. The body of our health data model consists of two sections: one entitled "medical equipment" to describe the sensor used $[13,14]$ and another section entitled "Vital signs measurement" to display patient's vital signs taken at a specific time. The "Medical equipment" section contains all the information required for identifying the sensor: the manufacturer's name, the model, the sensor type nomenclature, its identifier or its serial number. The "Vital signs measurement" section has one or more predefined entries according to the number of vital signs measured by the sensor $[13,14]$. Indeed, some medical equipment can analyze two vital signs or more (e.g. blood pressure and pulse rate for a blood pressure monitor) [15]. In such cases, the health data model has several entries in the same section which correspond to different measured vital signs. Each en-try also contains the date and time of the self-measurement, the measurement's type nomenclature code and the measurement unit. Moreover, some vital sign may require several measurements. In HL7 CDA document, all measurements required to obtain the vital sign are linked by the HL7 element "entry relationship" $[13,16]$.

At all stages of patient's data transfer, all data of the health context model must be communicated to the different receivers.

\subsection{Interoperability of the telemedicine systems}

To guarantee quality and safety of patient's care our telemedicine application combines three different systems: (1) the patient system for the medical monitoring and patient selfmeasurements, (2) the data processing and archiving system, which is generally located in the hospital and (3) the data transmission system between the two structures. These three systems describe the workflow of the patient's data from his home to the hospital and must be interoperable. Continua Health Alliance (CHA) is a non-profit organization that promotes standard data exchange formats, integration profiles and standard transport protocols. The Guidelines of the CHA [17] help to ensure interoperability between all the components of the three systems of our telemedicine architecture, based on international standards and Integrating the Health-care Enterprise (IHE) technical frameworks. To guarantee plug \& play capability, the CHA prescribes for the patient system the use of the ISO/IEEE 11073 standards family [17] for communication between medical devices and application hosting devices, such as mobile phones and set-top boxes. For the data transmission system, an IHE profile and the Health Level 7 (HL7) V2.6 [18] standard is used to transfer securely health data from patient's home to health professionals. The IHE DEC (Device Enterprise Communication) profile defined in the IHE PCD (Patient Care Device) technical frame-work allows a point-to-point communication between a hosting device and a remote server [19]. A HL7 v2.6 message is transmitted to the remote server based on the information contained in the hosting device. For the data processing and archiving sys-tem, IHE has proposed the XDS (Cross-enterprise Document Sharing) profile 


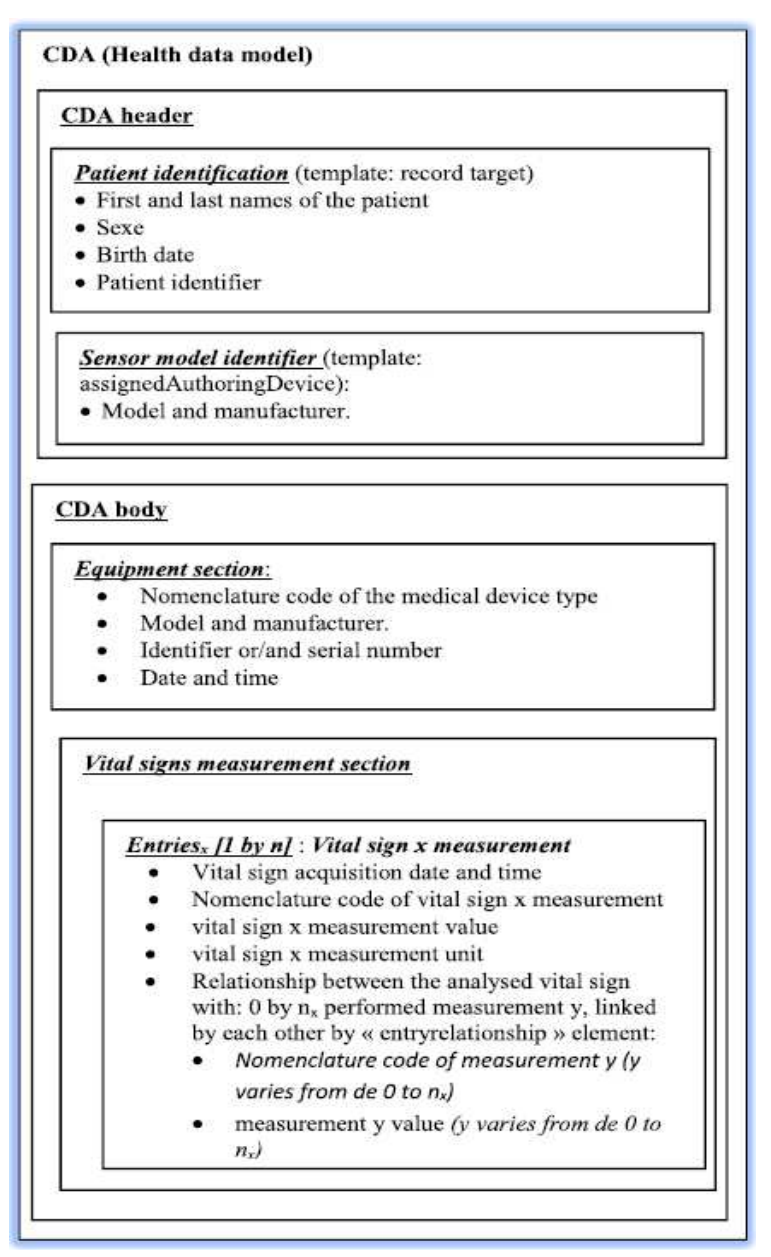

Fig. 2. The health context model of vital sign measurement.

[20], which comes from the IHE IT Infrastructure technical framework (ITI TF) [20]. This profile describes how to share medical documents appropriately. It specifies the transactions supporting the registration, distribution and access to shared documents between healthcare facilities [20]. Fig.3 summarizes the different steps of data transport by the three interoperable systems with the use of communication standards, standard health data exchange formats and integration profiles.

\subsubsection{Models and data structure based on the communication standards}

To represent data and to define data access, the ISO/IEEE 11073 family of standards provides models containing objects and attributes. They help us to describe the data reception and transfer processes from one system to another. The system model of the ISO/IEEE standard 11073-20601 is divided into three main components: the domain information model (DIM), the service model, and the communication model [21]. These objects and their attributes represent the elements that control the behavior of the

\subsubsection{The DIM model}

For the patient system interoperability and the trans-

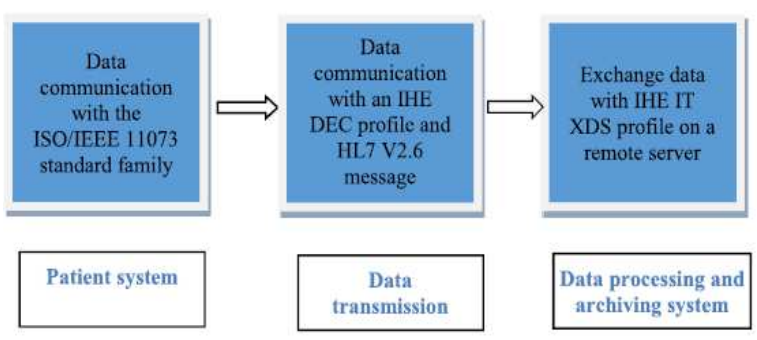

Fig.3. The data communication.

port of our data structure model, each of the installed sensors must support the ISO/IEEE 11073 standards. The first step is to describe the DIM to analyze its objects and attributes which number of physiological measurements to exchange and on the device status at the host device [22]. These components are essential items of the communication protocol between the sensor and the hosting device. The most important object is the Medical Device

System (MDS) [22]. It represents the properties and services of the medical device. The attributes of the MDS object allow to identify the device, to describe its technical characteristics and state. Besides the MDS object, there are Persistent Metric Store (PMstore) objects and other objects (zero or more per object type) which are derived from the metric class [22]. These different objects are numeric objects which represent episodic measurements, Real Time Sample Array (RT-SA) objects which represent continuous samples or waveforms, enumeration objects which represent event annotations and PMstore which provides a persistent storage mechanism [22]. The medical devices at patient's home do not necessitate internal storage memory. All vital signs are transmitted automatically after acquisition. Consequently, the "Persistent Metric Store" and the "Permanent metric segment" objects [22] are not required. The proposed generic DIM model for all sensors at patient's home comprises two types of objects: one MDS object and one or more numeric objects (depending on the number of measured vital signs: cf. Fig.4) [22].

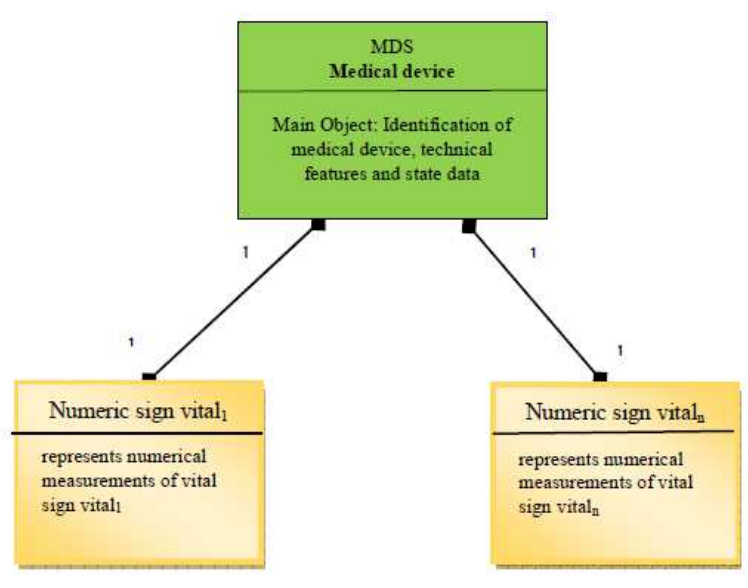

Fig. 4. DIM model for sensors at patient's home. 


\subsubsection{Models comparison}

To verify the first step of transport of all structured data of the medical device (data transport with the ISO/IEEE standards), we analyzed the relation between the DIM data model and our health context model. The aim of this study was to verify the appropriate mapping of all components between the two models (see Table1). The only difference between these models was the use of different nomenclature systems for medical terms. To achieve semantic interoperability of the telemedicine application, it was required to elaborate a mapping between the ISO/IEEE 11073-10101 numeric codes and the equivalent LOINC term(s) [22]. About sensor specific items, the DIM model from ISO/IEEE 11073 standards, the HL7 V2.6 standard and the HL7 CDA R2 standard [10a] all contain the following items: the manufacturer's name, the sensor model, a unique identifier and a serial number $[19,22,16]$.

All these data are communicated by the sensor itself [26].

\begin{tabular}{|c|c|c|c|c|}
\hline \multicolumn{2}{|c|}{ The health data model } & \multicolumn{3}{|c|}{ The DIM model } \\
\hline $\begin{array}{l}\text { Location field in the health } \\
\text { data model }\end{array}$ & $\begin{array}{c}\text { Item in the health data } \\
\text { model }\end{array}$ & Attribute name & Attribute Identification & $\begin{array}{l}\text { Location field in } \\
\text { the model DIM }\end{array}$ \\
\hline $\begin{array}{l}\text { CDA HEADER : } \\
\text { - } \underline{\text { Sensor model Identifier }}\end{array}$ & Model and manufacturer & System-Model & MDC_ATTR_ID_MODEL & $\begin{array}{l}\text { Attributes included } \\
\text { in MDS object }\end{array}$ \\
\hline $\begin{array}{l}\text { CDA body : } \\
\text { Equipment section: } \\
\begin{array}{l}\text { Nomenclature code } \\
\text { of the medical } \\
\text { device type }\end{array}\end{array}$ & $\begin{array}{l}\text { Nomenclature code of the } \\
\text { medical device type }\end{array}$ & System-Type & MDC_ATTR_SYS_TYPE & $\begin{array}{l}\text { Attribute included } \\
\text { in MDS object } \\
\text { (with nomenclature: } \\
\text { ISO/IEEE 11073- } \\
\text { 10101) }\end{array}$ \\
\hline $\begin{array}{l}\text { CDA body : } \\
\text { Equipment section: } \\
\begin{array}{l}\text { Model and } \\
\text { manufacturer }\end{array}\end{array}$ & Model and manufacturer & System-Model & MDC_ATTR_ID_MODEL & \multirow{3}{*}{$\begin{array}{l}\text { Attribute included } \\
\text { in MDS object }\end{array}$} \\
\hline $\begin{array}{l}\text { CDA body : } \\
\text { Equipment section: }\end{array}$ & \multirow{2}{*}{$\begin{array}{l}\text { Identifier or/and serial } \\
\text { number }\end{array}$} & System-Id & MDC_ATTR_SYS_ID & \\
\hline $\begin{array}{l}\text { - Identifier or/and } \\
\text { Serial number }\end{array}$ & & $\begin{array}{l}\text { Production- } \\
\text { Specification }\end{array}$ & MDC_ATTR_ID_PROD_SPECN & \\
\hline$\frac{\text { CDA body : }}{\frac{\text { Equipment section: }}{\text { - Date and time }}}$ & Date and time & Date and time & MDC_ATTR_TIME_ABS & $\begin{array}{l}\text { Attributes included } \\
\text { in MDS object }\end{array}$ \\
\hline
\end{tabular}

The vital sign requires only one measurement

\begin{tabular}{|c|c|c|c|c|}
\hline $\begin{array}{l}\text { CDA body : } \\
\text { - Vital signs measurement } \\
\text { section: Entries } x_{x}\lfloor\text { by } n\rceil\end{array}$ & $\begin{array}{c}\text { Date and time of the } \\
\text { measurement }\end{array}$ & $\begin{array}{l}\text { Absolute-Time- } \\
\text { Stamp }\end{array}$ & MDC_ATTR_TIME_STAMP_ABS & $\begin{array}{c}\text { Attribute included } \\
\text { in numeric sign } \\
\text { vital }_{\mathrm{x}} \text { object defines } \\
\text { the date and time of } \\
\text { observation }\end{array}$ \\
\hline $\begin{array}{l}\frac{\text { CDA body : }}{\text { - Vital signs measurement }} \\
\frac{\text { section: } \text { Entries }_{x}[1 \text { by } n]}{\text { Nomenclature code of }} \\
\text { vital sign }{ }_{x} \\
\text { measurement }\end{array}$ & $\begin{array}{l}\text { Nomenclature code of } \\
\text { vital sign x measurement }\end{array}$ & Type & MDC_ATTR_ID_TYPE & $\begin{array}{l}\text { Code included in } \\
\text { numeric sign vital } \\
\text { object (with } \\
\text { nomenclature: } \\
\text { ISO/IEEE 11073- } \\
\text { 10101) }\end{array}$ \\
\hline 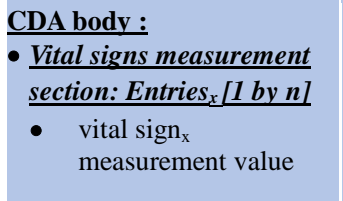 & Measurement value & $\begin{array}{c}\text { Simple-Nu- } \\
\text { Observed-value } \\
\text { or Basic-Nu- } \\
\text { Observed-value } \\
\text { or Nu-Observed- } \\
\text { value }\end{array}$ & $\begin{array}{c}\text { MDC_ATTR_NU_VAL_OBS_SIMP } \\
\text { or } \\
\text { MDC_ATTR_NU_VAL_OBS_BASIC } \\
\text { or MDC_ATTR_NU_VAL_OBS }\end{array}$ & $\begin{array}{l}\text { Attribute included } \\
\text { in numeric sign } \\
\text { vital }_{\mathrm{x}} \text { object defines } \\
\text { the measurement } \\
\text { value. }\end{array}$ \\
\hline $\begin{array}{l}\text { CDA body : } \\
\text { - Vital signs measurement } \\
\frac{\text { section: Entriesx [1 by } n]}{-\quad \frac{\text { vital signx }}{\text { measurement unit }}}\end{array}$ & Measurement unit & Unit-Code & MDC_ATTR_UNIT_CODE & $\begin{array}{l}\text { Code included in } \\
\text { numeric sign vital } \\
\text { object (with } \\
\text { nomenclature: } \\
\text { ISO/IEEE 11073- } \\
\text { 10101) }\end{array}$ \\
\hline
\end{tabular}

Table1: Comparison between the health context model and DIM. 


\begin{tabular}{|c|c|c|c|c|}
\hline \multicolumn{5}{|c|}{ The vital sign requires several measurements } \\
\hline $\begin{array}{l}\text { CDA body : } \\
\text { Vital signs measurement } \\
\frac{\text { section: }[1 \text { by } n}{\text { - Date and time of the }} \\
\quad \text { vital sign }{ }_{\mathrm{x}} \text { measurement }\end{array}$ & $\begin{array}{l}\text { Date and time of the } \\
\text { measurement }\end{array}$ & $\begin{array}{l}\text { Absolute-Time- } \\
\text { Stamp }\end{array}$ & $\begin{array}{c}\text { MDC_ATTR_ID_ABS et } \\
\text { MDC_ATTR_TIME_STAMP_ABS }\end{array}$ & $\begin{array}{l}\text { Attribute included } \\
\text { in numeric sign } \\
\text { vital }_{x} \text { object }\end{array}$ \\
\hline $\begin{array}{l}\text { CDA body : } \\
\text { - Vital signs measurement } \\
\text { section: Entries }{ }_{x}[1 \text { by } n] \\
\text { - } \begin{array}{l}\text { Date and time of the } \\
\text { vital sign }{ }_{x} \text { measurement }\end{array}\end{array}$ & $\begin{array}{l}\text { Nomenclature code of vital } \\
\text { sign } \mathrm{x} \text { measurement }\end{array}$ & Type & MDC_ATTR_ID_TYPE & $\begin{array}{l}\text { Code included in } \\
\text { numeric sign vital }_{\mathrm{x}} \\
\text { object (with } \\
\text { nomenclature: } \\
\text { ISO/IEEE 11073- } \\
\text { 10101) }\end{array}$ \\
\hline $\begin{array}{l}\text { CDA body : } \\
\text { Vital signs measurement } \\
\text { section: Entries } \underline{x}[\mathbf{1} \text { by } n] \\
\text { - Relationship between the } \\
\text { analysed vital sign with: } \\
0 \text { by } \mathrm{n}_{\mathrm{x}} \text { performed } \\
\text { measurement y, linked by } \\
\text { each other by } \\
\text { « entryrelationship » } \\
\text { element: } \\
\text { - Nomenclature code of } \\
\text { measurement y }(\mathrm{y} \\
\left.\text { varies from de } 0 \text { to } \mathrm{n}_{\mathrm{x}}\right) \\
\text { - measurement y value }(\mathrm{y} \\
\text { varies from de } 0 \text { to } \mathrm{n}_{\mathrm{x}}\end{array}$ & $\begin{array}{l}\text { List of different } \\
\text { Nomenclature codes of the } \\
\text { different measurements to } \\
\text { obtain the vital signs }\end{array}$ & Metric-Id-List & MDC_ATTR_ID_PHYSIO_LIST & $\begin{array}{l}\text { Attribute included } \\
\text { in numeric sign } \\
\text { vital } \text { x }_{\mathrm{x}} \text { object. This } \\
\text { attribute is used } \\
\text { when a compound } \\
\text { value is used. The } \\
\text { order of the metric- } \\
\text { Id-List is the same } \\
\text { than the order of the } \\
\text { compound observed } \\
\text { value }\end{array}$ \\
\hline $\begin{array}{l}\text { CDA body : } \\
\frac{\text { Vital signs measurement }}{\text { section: Entries }}{ }_{x}^{[1 \text { by } n]} \\
\begin{array}{l}\text { vital } \text { sign }_{x} \text { measurement } \\
\text { unit }\end{array}\end{array}$ & Measurement unit & Unit-Code & MDC_ATTR_UNIT_CODE & $\begin{array}{l}\text { Code included in } \\
\text { numeric sign vital }_{\mathrm{x}} \\
\text { object (with } \\
\text { nomenclature: } \\
\text { ISO/IEEE 11073- } \\
\text { 10101) }\end{array}$ \\
\hline
\end{tabular}

Table1 (continued)

\subsection{HL7 V2.6 message}

To transfer the health context model data from the patient's home to a remote location, the IHE DEC profile advises the use of a HL7 v2.6 message which contains patient's information in encoding syntax based on segments [19]. This type of message has two main characteristics: it can be sent without solicitation from the remote location and its structure covers all observations and results which come from the patient's home. The name of the message type is "ORU^R01^ORU_R01" [19]. It consists of several optional or mandatory data segments: the Message Header (MSH), The Observation Result segment $(\mathrm{OBX})$ and the Observation Request segment (OBR). The MSH defines the identification of message, the sender and receiver, the message type, and some specifics of the syntax of a message [19]. The OBX is used to transmit a single observation or observation fragment [23]. All results of patient's measurements should be contained in OBX segments. The third component is the observation Request segment (OBR). It contains the re-quest of the medical act (ordering information) and the context for observation (i.e., whether the request was done in-clinic or remote). When a set of observations is ordered, the order message contains an OBR segment [23]. Some fields in the OBR segment only apply to the ordering message.

For remote monitoring systems, the message structure of "ORU^OR01^ORU^R01" is suitable to transmit data between the patient's home and a remote health care facility [19]. In the message header, the patient's identification and contact information may be transmitted in the Patient Identification (PID) segment. All results of patient's measurements are contained in OBX segments. The identification of the device will be present in an OBX segment. In this case, the suffix of the OBX-3-observation identifier is DEV [23]. In the OBR-4 of the OBR segment, we have the identifier codes for the requested patient's measurements. For the transfer of the message, it is advised to use a secure standard protocol such as HTTPS [19]. The HL7 message sent from the patient's home allows the patient's data to be processed and stored on a remote server. When a patient's measurement threshold is exceeded, the data processing and archiving system must be able to generate and display alerts or to indicate the actions to be implemented in case of emergency. In this case, a CDA document can be created. 


\subsection{CDA document}

The CDA document can be automatically generated by an information system and made accessible by medical professionals. The document sharing management system can be built with the XDS profile functional architecture [20]. This profile describes appropriate sharing of medical documents. It specifies the transactions supporting the registration, distribution and access to shared documents between healthcare facilities [20]. With the IHE XDS profile, sending and receiving clinical documents can be achieved with standard communication protocols as HTTP or HTTPS [24]. The created CDA documents consist of a header and a body, that include structured elements in both parts of the CDA documents. With the CDA R2, the body may contain health encoded data that can be easily integrated into databases of healthcare facilities. This ensures the interoperability between clinical systems and data integration of all parts of our proposed health data model. For implementation of a CDA document, we have selected an existing model that contains templates, rule sets and value sets for vital signs: PHMR [13]. The implementation guides for CDA [13-15] contain templates that address specific uses. They can represent professional recommendations, or national clinical practice guidelines.

To optimize our model, we selected an implementation guide with minimal CDA structure "Minimal structuration of medical documents" [25]. For the proposed CDA document header, it is required to add only one template "assigned Authoring Device" for the identification of the sensor model [13-15]. As with the health model data, the proposed CDA document consists of two sections. One of which is called "Medical Equipment" and the other "Patient vital signs" [13,14]. The "Medical Equipment" section contains the sensors characteristics described in the health data model $[13,14]$. In the "Patient vital signs" section, each measured vital sign is introduced as a predefined entry. All measurements required to obtain the vital sign are described ac-cording to the "observation" template [13-15].

\section{Experiments in progress}

Laboratory experimentations have enabled to validate this health data model for the following sensors: a blood pressure monitor UA-767PBT, A\&D Company Ltd, a pulse oximeter 3150 Wirstworn, Nonin Medical Inc, and a weighing scale UC-321PBT, A\&D Company Ltd. They are all CHA certified ${ }^{2}$.

These three sensors provide all possible configurations of our health data model: the weighing scale DIM model has only one numeric object,

\footnotetext{
2 The list of CHA certified medical devices is presented on the following website: http://www.pchalliance .org /certified -product showcase.
}

whereas the blood pressure monitor DIM model and the pulse oximeter DIM model have two numeric objects.

For the weighing scale and the pulse oximeter, only one measurement is sufficient to obtain the vital sign. By contrast, the blood pressure vital sign is composed of several measurements (the systolic and diastolic blood pressure measurements). The three sensors are provided by two different manufacturers (A\&D Company Ltd and Nonin medical). In a laboratory trial, an open source implementation Antidote software [9] provides an opportunity to achieve interoperability between the sensors and the smartphone which are compliant with the ISO/IEEE 11073 family of standards [9].

Various communication tests of vital signs have been conducted with the three sensors to prove the interoperability of the patient system. We also checked the data completeness for a proper remote interpretation. To test the data transfer to a re-mote system, we used a function of the Antidote software [9] which allows to send the patient's data by e-mail. To test the medical data exchange, we have developed one HL7 CDA document which collects several vital signs. We have tested this document validity with the Gazelle platform tool [10]. The first results of the experiments prove that implementing interoperable infrastructure for the three systems of our telemedicine application is achievable.

\section{Discussion}

A fully interoperable infrastructure of a telemedicine system and health data model provides a Telemedicine Systems Interoperability Framework for the monitoring of chronic diseases. They cover the needs of patients suffering from one or several chronic diseases. Currently, only research work on the relationship between the DIM of the IEEE standard 11073 family and the RIM which is the source of CDA have been done [27]. These works contribute to achieve an interoperable telemedicine system. They enable data transmission from only a medical device (internally-implanted device: a cardiac defibrillator, or a pacemaker) to a medical application software by sending a HL7 V3 message. To build the message HL7 V3 con-tent, the link between both models (DIM and RIM) allows to get data from the implanted device with ISO IEEE 11073 standards.

Our research provides an interoperable infrastructure of a telemedicine system at patient's home for chronic disease monitoring. The list of usable medical devices at patient's home corresponds to the equipment described in specialization standards of the ISO/IEEE 11073 (ISO/IEEE 11073-104zz) [22]. All these medical devices address the needs of patients for several chronic diseases. Despite this, this list contains only simple devices. No medical device is able to deliver medical wave-forms, such as electrocardiogram for chronic heart failure or spirometry waveform for chronic respiratory failure. 
Likewise, our proposed health data model is not suitable for this type of medical device. In fact, the DIM of this medical device type [28] is different and includes other objects for modeling of the real-time collection of data. To be close to real-time communication, these devices must in specific cases continuously send data. In such cases, they must be equipped with alarm systems. Alarms are thus generated if the curve exceeds one or several thresholds fixed by the medical team.

IHE proposed a trial implementation about the inclusion of waveform data in IHE PCD profiles: Waveform Content Module (WCM). This technical framework supplement describes a method near real time to send waveform data through HL7 V2 messages [29]. This guide also promotes WCM model for the description of the data structure and semantics to be used for communication of waveforms which follows the IEEE 11073 DIM. It provides attributes which are similar to that of the IEEE 11073 Real Time SA object [29]. They are used to describe, for example, the waveform type, the curve settings, such as sampling rate, resolution, encoding and data range and information on alarms. To be able to transmit waveforms, the health data model of medical devices must contain the same information as the WCM model [29].

For the communication of medical waveforms, the medical equipment type requires another patient system configuration. In fact, to be close to real-time communication, medical devices must have the ability to send a message approximately every second [29]. In this case, it is necessary to have broad-band access to avoid the network congestion and to have a speed of at least $128 \mathrm{kbit} / \mathrm{s}$ [30]. As the minimal speed is $384 \mathrm{kbit} / \mathrm{s}$, a standard broadband network (such as ADSL or $3 G$ ) meets the requirements of the patient system for the communication of medical waveforms. On the other hand, these medical devices must also have additional modules for the reception and processing of the patient's data. They must be equipped with alarm systems. Alarms are thus generated if the curve exceeds one or several thresholds fixed by the medical team. Furthermore, such facilities are costly to introduce.

Depending on the level of patient disease, real-time remote continuous monitoring can improve through the early detection of worsening of the disease and symptoms. For example, to detect cardiac arrhythmias, some continuous cardiac monitoring can provide automatic wireless transmission of abnormal ECG waveforms at the time of event, from the patient's home [31]. This system for the most high-risk patients can reduce the risk of sudden death (with the implantation of a pacemaker or cardiac defibrillator) [31]. The problem is the lack of interoperability of this system with other medical devices and hospital information systems. The use of WCM data model is to improve the integration of this medical device type in a technology platform in patient's home. The increase of home care should contribute to a continuation of this work.

\section{Conclusion and perspectives}

Telemonitoring is a key element for at home preventive care of vulnerable population with chronic health conditions. The data collected at the patient's home must be exhaustive to sup-port adequate interpretation and follow up of vital signs and medical data exchange between healthcare professionals. We propose an implementation of a health data model to support adequate interpretation and to facilitate medical data exchange between healthcare professionals. We have modeled an inter-operable solution for the three systems of telemedicine applications. This ecosystem promotes the use of the ISO/IEEE 11073 standards family for communication between medical devices and computer systems, and the use of HL7 standards and of IHE integration profiles for exchanging clinical data among information systems. To extend the implementation of most complex medical equipment, the proposed health data model of medical devices must contain other modules to describe the new functions.

\section{Conflict of interest statement}

The authors have not declared any conflicts of interest.

\section{Acknowledgements}

We would like to thank Dr Mohammad HAYDAR, cardiologist in Alençon, for his willingness to take part in this study, the AZNnetwork company for its participation in experimentation and Eric Poiseau (INRIA) for his assistance in the choice of CDA implementation and test tools.

\section{References}

[1] Finet P, Le Bouquin Jeanne R, Dameron O, Gibaud B. Review of current telemedicine applications for chronic diseases. Toward a more integrated system? IRBM March 2015;36(3):133-57.

[2] World Health Organization. Global status report on non communicable diseases; 2011.

[3] United Nations. World population prospects: the 2006 revision. New York: United Nations, Department of Economic and Social Affairs, Population Division; 2007. Available from: http://www.un.org/esa/population/publications/wpp2006/WPP2006 _Highlights_rev.pdf. [Accessed8November2013].

[4] Mathers CD, Bernard C, Melgaard K, et al. Global burden of disease in 2002: data sources, methods and results. In: Global programmed on evidence for health policy discussion paper No. 54 . Geneva: World Health Organization; 2003.

[5] Martín-Timon I, Sevillano-Collantes C, Segura-Galindo A, Javier del Camilo-Gómez F. Type 2 diabetes and cardiovascular disease: have all risk factors the same strength? World J Diabetes 2014 August 15;5(4):444-70.

[6] Craft RL. Toward technical interoperability in telemedicine. Telemed J E-Health 2005;11(3):384-404.

[7] Baas M, Bernonville S, Proconsul N, Hassler S, Koski C, Boy AG. To-wards a context model for human-centered design of contextual data en-try systems in healthcare domain. In: 16th international conference on human-computer interaction, Heraklion, Crete, Greece, June 22-27, 2014, pp.223-33. 
[8] Dolin RH, Alschuler L, Beebe C, Biron PV, Boyer SL, Essen D, et al. The HL7 clinical document architecture. J Am Med Inform Assoc 2001; 8:552-69.

[9] Aravind S, Natarajan V. Developing software for IEEE 11073 device specializations using antidote library. Int $\mathrm{J}$ Eng Innov Technol Apr. 2016;5(10)

[10] Boufahja A, Parizeau E, Thomazon G, Barge A. Model-based analysis of HL7 CDA R2 conformance and requirements coverage. Eur J Biomed Inform January 2015;11(2):41-50.

[11] Ferranti JM, Musser RC, Kawamoto K, Hammond WE. The clinical document architecture and the continuity of care record: a critical analysis. J Am Med Inform Assoc May-January 2006; 13(3):245-52.

[12] Health Level Seven. HL7 implementation guide: S\&I framework transitions of care companion guide to consolidatedCDA for meaningful use stage 2, release 1 - US realm. Available from: https://www.hl7.org /implement/standards/product brief .cfm?product_id =258. [Accessed7 November 2015].

[13] Health Level Seven. HL7 implementation guide for CDA release 2: Personal Healthcare Monitoring Report (PHMR), release 1.1, October 2010

[14] Health Level Seven. HL7 implementation guide: CDA release 2 - Continuity of Care Document (CCD), standard specification for Continuity of Care Record (CCR), April 2007.

[15] ASIP Santé. Cadre d'Interopérabilité SIS - couche contenu volet modèle de contenu CDA. Available from: http:// esante.gouv.fr /sites /default /files /CI -SIS_CONTENU_VOLETSTRUCTURATIONMINIMALE_V1.3.1.1.pdf.

[Accessed29June2017].

[16] Dolin RH, Alschuler L, Boyer S, Beebe C, Behlen FM, Biron PV, et al. HL7 clinical document architecture, release 2. J Am Med Inform Assoc January-February 2006; 13(1):30-9.

[17] WartenaF, Muskens-SchmittL. Continua: the impact of a personal telehealth ecosystem. In: International conference on eHealth, telemedicine, and social medicine - etelemed; 2009. p.13-

[18] Frohner M, Urbauer P, Forjan M, Pohn B, Gerbovics F, Sauermann S, et al. Development of an Android app in compliance with the continua health alliance design guidelines for medical device connectivity in health. Biomed Tech September 6, 2012; 57(1).

[19] IHE. IHE patient care device (PCD) technical framework, vol.1 PCD TF-1 integration profiles; 2013 October 11.

[20] Duftschmid G, Rinner C, Kohler M, Huebner-Bloder G, Saboor S, Ammenwerth E. The EHRARCHE project: satisfying clinical information needs in a shared electronic health record system based on IHE XDS and archetypes. Int $\mathbf{J}$ Med Inform December 2013; 82(12):1195-207.

[21] Korsakov IN, Kuptsov SM, Manometer DA, Felsite VV, Sumiko MA. On demand plugins for X73-PHD manager. Int J Adv Trends Comput Sci Eng 2013; 5:35-40.

[22] IEEE. Stud 11073-20601: 2010 health informatics - personal health device communication - part 206101: application profile optimized ex-change protocol; 2010 May 1.

[23] Health Level Seven International (HL7). The HL7 version 2.6 standard; definitive version; October 2007.

[24] IHE International Inc. IHE IT infrastructure (ITI) technical framework volume 1 (ITI TF-1) integration profiles revision 10.1 final text; October 2011.

[25] ASIP Santé. Cadre d'Interopérabilité des SIS couche contenu, volet structuration minimale de documents médicaux. Version 1.3.1.1; December 2012

[26] Continua Health Alliance. Continua design guidelines, version 2016. Available from: https://cw.continuaalliance.org/document/dl /p699. [Accessed20 November 2016].

[27] Yuksel M, Dogac A. Interoperability of medical device information and the clinical applications: an HL7 RMIM based on the ISO/IEEE 11073 DIM. IEEE Trans Inf Technol Biomed 2011 July:15(4):557-66.

[28] IEEE. Std 11073-10201 health informatics - point-of-care medical device communication - part 10201: domain information model; 2005 December 15.

[29] IHE. IHE patient care device (PCD) technical framework supplement, waveform content module (WCM), trial implementation; 2012 August 16.
[30] Norris TE, Hart GL, Larson EH, Tarczy-Hornoch P, Masuda DL, Fuller SS, et al. Low-bandwidth, lowcost telemedicine consultations in rural family practice. J Am Board Fam Pract 2002; 15:123-7.

[31] Anderson J, Donnelly N. New methodologies in arrhythmia monitoring. Eur Cardiol 2008; 4(2):63-6. 